\title{
From Foundation Stones to Life: Concepts and Results
}

\author{
Marie-Christine Maurel $^{1}$ and Fabrice Leclerc $^{2}$
}

In tribute to James P. Ferris of Rensselaer Polytechnic (USA) - A pioneer in 'origin of life' research

\begin{abstract}
Systems consisting of mineral surfaces, water, salts and organic molecules are considered to be plausible models of early Earth's prebiotic environments. The probable involvement of clays, highly soluble minerals, sulfides and other minerals at the beginning of life have spurred a number of experimental studies to investigate organic molecule adsorption, polymerization and catalytic reactions of relevance to prebiotic chemistry. This article reviews current ideas in how life originated, summarises experimental results and presents some of the existing challenges that still beset the field of the origins of life.
\end{abstract}

KEYWORDS: mineral, salts, hydrothermal sites, extremophile, RNA, origins of life

\section{INTRODUCTION}

Researchers studying the origin of life are trying to determine the path that the building blocks of life took in developing biological functions: from synthesizing the initial building blocks to the emergence (through organization and evolution) of functional biomolecules such as those responsible for genetic replication and metabolism, which are two essential characteristics of life as we know it. In modern organisms, enzymatic proteins are required for the activation of nucleotide monomers (by phosphorylation), for the condensation of monomers into oligomers (molecules made of a small number of monomer units up to a few dozen), and for

\footnotetext{
${ }^{1}$ Institut de Systématique, Evolution, Biodiversité (ISyEB), CNRS, MNHN, UPMC, EPHE, UPMC, Sorbonne Universités, F-75005 Paris, France

E-mail: marie-christine.maurel@upmc.fr

${ }^{2}$ Institute for Integrative Biology of the Cell (I2BC)

Dept. of Genome Biology, CEA, CNRS, Université Paris Sud

Gif-sur-Yvette, F-91198, France

E-mail: fabrice.leleclerc@i2bc.paris-saclay.fr
} 
the replication of DNA. But such enzymatic proteins are themselves encoded in the genetic system. So, the question of which appeared first on Earth-the genetic information or the metabolism-is a chicken-and-egg dilemma. In the early 1980s, the discovery of ribozymes (short RNA sequences that bear catalytic activity) provided a new perspective on this dilemma and spawned the idea of a genetic world made exclusively of RNA that could have preceded the contemporary DNA/RNA/protein genetic world. However, replicating RNA would have been very difficult prebiotically, because each stage in the replication process requires enzymatic catalysis.

When discussing possible prebiotic scenarios for the origin of life, it is widely believed that mineral surfaces played an important role in adsorbing organic molecules and acting as a template to catalyze specific reactions. There are two reasons for this assumption. First, because of the hydrolytic power of water, any organic molecules synthesized would have been subsequently destroyed and the "prebiotic soup" would have been, in fact, a rather diluted thin soup (solution) that contained less than $10^{-4} \mathrm{M}$ carbon (Miller 1987). The probability of random collisions between relevant molecules in primitive bio-organic reactions would have been very low in such a dilute aqueous solution. It seems more realistic to assume that relatively high concentrations of organic substances were reached in systems isolated from the ocean): these could include lagoons, moist soil, and both terrestrial and oceanic hydrothermal sites that would be subject to temperature gradients and hydration-dehydration cycles. Molecules would also be brought into close contact with mineral surfaces in such environments.

A second rationale for the role of minerals is that several of them are known to possess catalytic properties: some can generate reactive oxygen species (free radicals) at their surface when irradiated by ultraviolet light. These free radicals could have either catalyzed the formation or the destruction of complex organic molecules, depending on the specific reaction. Furthermore, and, alternatively, adsorption of organic molecules in the interlayers of minerals, such as clays, could have played a protecting role against decomposition of organic molecules by light. It is important to note thatminerals have been identified to form various prebiotic 
organic molecule polymers, but the relative abundance of these minerals and their extent of occurrence on early Earth, is highly questionable (see Schoonen et al. 2016 this issue).

In this article, we provide an overview of the current knowledge of prebiotic organic molecule polymerization at mineral surfaces under environmental conditions that may have existed on early Earth. The emphasis is on ribonucleic acid (RNA) oligomerization, because that will provide the route for the emergence of the genetic code. The four building blocks of RNA (i.e. the nucleotides of RNA) are made of the two purine nitrogenous bases of adenine $(A)$ and guanine $(G)$ and the two pyrimidine nitrogenous bases of cytosine (C) and uracil (U). Despite the massive body of work devoted to research on RNA polymerization, the mechanisms of nucleotide synthesis are still not understood. In the past few years, the "RNA world" paradigm has been challenged. New ideas have arisen that suggest the existence of a pre-RNA world where amino acids and peptides (compounds consisting of two or more amino acids linked in a chain) may have shaped the RNA world through, for example, the emergence of a simpler genetic system before the transition to modern genetic systems. In addition to RNA polymerization, we also address amino acid oligomerization to form peptides, something that would eventually evolve into enzymes for metabolic activity; the formation of hybrid RNA/amino acid and peptide/RNA nucleotide molecules; and the potential emergence of viroids before peptide enzymes appeared.

\section{MINERALS: FROM CATALYSIS TO METABOLISM}

One of the most challenging problems in the origin of life field is to explain the prebiotic synthesis of RNA nucleotides (monomers) from their basic components (nucleobase, ribose, and phosphate) and explain the respective activations that promote their polymerization under prebiotic conditions in the absence of enzymatic catalysis. But progress has been made to link the pre-RNA world to the RNA world. 


\section{Nucleotide Monomer Synthesis}

Saladino et al. (2007) showed that the four nucleobases (adenine, guanine,cytosine, and uracil) could be synthesised by heating formamide in the presence of mineral catalysts and ultraviolet (UV) light.

Ricardo et al. (2004) focused on the stabilisation of ribose (the carbohydrate sugar molecule that forms part of the backbone of RNA) by the borate minerals ulexite and colemanite. Akouche et al. (2016) later demonstrated how ribose could adsorb and be stabilized on amorphous silica, protecting the ribose sugar from degradation and significantly increasing the proportion of ribofuranose compared to ribopyranose forms (Georgelin et al. 2015). Like other building blocks of RNA, the source of ribose might also come from the interaction of cometary ices containing organic residues $\left(\mathrm{H}_{2} \mathrm{O}, \mathrm{CH}_{3} \mathrm{OH}, \mathrm{NH}_{3}\right)$ and space radiation (Meinert et al. 2016).

The occurrence of phosphate (the other piece of the RNA backbone), however, has been less discussed. It is generally admitted, but without any conclusive evidence, that phosphate sources were present on the early Earth. Where this phosphate originated is a crucial question because of the significant role that phosphate has in the structureof nucleic acids, in the coenzymes that are necessary for enzyme function, and in several metabolic processes. Phosphate is seen as the "molecular unit of currency" for energy transfer. Natural phosphides found in several types of rocks may be the origin of the reactive prebiotic phosphorus that was required on the early Earth (Britvin et al. 2015).

Although the three basic components of RNA could have been present in early Earth conditions and could be assembled into nucleotide monomers, the presence of specific peptide precursors (such as diamino-butyric acid) found in meteorites suggests an alternative path for the transition between the pre-RNA world (with unpolymerized nucleobases) to the modern genetic systems (with polymerized nucleobases). In the peptide model, nucleobases are built upon a peptide backbone instead of the phosphate-ribose backbone currently found in RNA. The condensation of nucleobase acetic acids and N-(2-aminoethyl)glycine (AEG), which are compounds identified in the "prebiotic soup" (Miller 1987), leads to the 
formation of a peptide nucleic acid (PNA) monomer units which could be later polymerized.

\section{Minerals as Catalysts}

Identifying the main organic catalysts (e.g. primitive enzymes) that contributed to the emergence of the earliest biochemical pathways is a major challenge to originof-life researchers (Maurel and Ninio 1987). Organic chemists have estimated that if two reactants are immobilized in a favourable position, the increase in the reaction rate through entropy effects may be as high as $10^{8}$. There is little doubt that the primitive enzymes were rigid, template-like structures that could adsorb the reactants, thereby decreasing the entropy factor of the chemical reactions. In such primitive, rigid and poorly specific systems, the efficiency of catalysis would have been solely dependent on the orientation and positioning of the reactants. Moreover, catalysis through entropy effects has a major shortcoming: the templatelike rigid catalyst cannot be as efficient as most modern enzymes. Nonetheless, even though inefficient, these primitive rigid catalysts (enzymes) could have arisen by chance events. It is logical to consider that mineral particles could behave very similarly to primordial enzymes by adsorbing, and thereby positioning, one reactant molecule with respect to the other. Minerals may also contribute to the condensation of prebiotic molecules and so help in the polymerization of these molecules.

Considering the range of minerals that were present in early Earth, clays are often considered as chemical mini-reactors in prebiotic synthesis. Clays are aluminosilicate minerals with a layered structure in which the plates stack like the pages of a book. Clay minerals carry an excess negative charge in their inter-layer sites as well as charges on the edges of the plates. In many clays, water and cations usually occupy the interlayer sites. Organic molecules can also be adsorbed in the interlayer sites or at edges of the clay plates Cclays can play a role as an ionexchanger. Some clays (e.g. montmorillonite) can swell and expand enormously in the presence of water and then collapse back upon dehydration. This combination of charged surfaces and swelling capability can concentrate organic molecules, creating an environment favorable for the catalysis of reactions between organic 
molecules and condensation of prebiotic molecules in geological environments that undergo hydration-dehydration cycles. Therefore, clays (such as montorillonite) have been used in a number of prebiotic chemical studies. For example, amino acidphosphate anhydride monomers can be condensed in the presence of minerals When the energy-rich anhydride bond in the monomer (SCHEME 1) is hydrolyzed, energy is released which enables the subsequent formation of bonds between amino acids, resulting in peptides. Nevertheless, one may question whether the C-O-P bond in the monomer could itself have formed without the presence of some catalyst.

Other experimental studies have focused on the adsorption and reaction of amino acids and nucleotide acids on clays (Paecht-Horowitz 1978; Ferris 2005). For example, the strong adsorption of nucleotides on montmorillonite is followed by the formation of a nucleotide dimer. Also studied is the regio-specificity (exclusive preference) of the phosphate-ribose linkages in RNA. It was shown that heterogeneous oligocytidylates were formed by the montmorillonite-catalysed condensation of activated monomers. The oligocytidylates could then serve as templates for the synthesis of the complementary strand of oligoguanylates.

\section{Synthesis of Long Polymers of Amino Acids and Nucleotides}

Most theories concerning biological organization assume that nucleotide polymers with lengths in the range of 30-100 monomers are sufficient to make a genetic system viable. As stated by Hill et al. (1998): “... long polymers were synthesized on the surface of minerals in a prebiotic process analogous to solid-phase synthesis." Their experimental study reported elongation and oligomerization of negatively charged amino acids adsorbed on hydroxylapatite and illite, as well as long oligopeptides of beta-glutamic acid on the surface of hydroxylapatite (Hill et al. 1998). Ferris et al. (1996) showed that montmorillonite could induce the formation of nucleotide oligomers up to 55 monomers long and that illite or hydroxylapatite promoted peptide oligomer formation up to 55 amino acids long. In both of these studies, oligomerization of the nucleotides and amino acids on mineral surfaces was achieved by successive additions (or "feedings) of fresh monomers of the respective nucleotides and amino acids. 
Catalysis by minerals is unique: the type of catalytic specificity brought about by a particular mineral surface cannot be assumed to extend generally to all metabolites. If non-specificity were the case, then we would have to search for multifunctional surfaces, which are highly unlikely to find. These observations suggest that the complete suite of catalytic mineral surfaces for promoting different types of prebiotic reactions would have to have been available on the primitive Earth. While many studies have reported long oligomerization of nucleotides, Burcar et al. (2013) argued that a single method of identifying oligomers may not produce reliable results.

The next step in investigating the potential roles of minerals might be to consider the possibility of mineral surface-bound replicating systems as precursors to template-directed reactions of nucleotides. This kind of study has not been reported in the literature to date but provides an interesting angle for future investigations., Mathematical modelling suggests that a template-directed ligation mechanism may operate to facilitate this transition where the ratio between ligation and breakage rates should be $>1$ at concentrations $<1 \mathrm{M}$ (Tkachenko and Maslov 2015). This model opens new perspectives to experimentally explore the formation of long polymers at low nucleotide concentrations.

Protocell-like vesicle self-assembly can be catalyzed by many minerals, including clay surfaces (Szostak 2012). In a further conjectured step towards a cellular life, a possible clay-related scenario involves RNA molecules, formed by clay-catalyzed polymerization, being incorporated within protocell-like vesicle membranes. The switch from a surface-bound to a surface-free RNA-based replicating system could then be possible through the emergence of derived (auto)catalytic RNAs or ribozymes. These proposals provide directions for future research (Szostak 2012).

\section{Evaporites: the Role of Soluble Minerals and Inorganic Ions in RNA Stability}

Primordial life may have originated in hypersaline conditions because it is likely that primitive oceans on early Earth were highly saline. Salt is also abundant on the planet Mars and on Jupiter's moon Europa. Extremely halophilic Archaea are organisms that require very high salt concentrations for their survival. They occur in salt flats and evaporitic lakes, such as the Dead Sea in the Middle East or the Great 
Salt Lake (Utah, USA). These halophile organisms compensate for the high osmotic pressure of their environment by accumulating levels of $\mathrm{K}^{+}$and $\mathrm{Cl}^{-}$close to saturation with respect to sylvite $(\mathrm{KCl})$ in their cytoplasm. And very ancient salttolerant micro-organisms have been isolated from brine inclusions in rock salt crystals. Halophiles may not have appeared as the earliest organisms but their proteins have evolved particular features of halo-adaptation, which provide them a great tolerance to decreased water activity and to temperature variations. This makes them good candidates for biological traces of survival in dry ancient environments.

Hypersaline conditions can stabilize organic molecules and promote polymerization. For example, the structural integrity of transfer RNA (tRNA) can be maintained at high temperatures (e.g. $82{ }^{\circ} \mathrm{C}$ for $30 \mathrm{~h}$ ) and high salt concentrations (e.g. $2 \mathrm{M} \mathrm{NaCl}$ ). The tRNA adopts a specific cloverleaf 2-D structure (FIG. 1A) and an L-shaped 3-D structure (Fig. 1B). These structures can be further stabilized by metal ions (e.g. $\mathrm{Mg}^{2+}, \mathrm{Ca}^{2+}, \mathrm{Mn}^{2+}$ ), which are generally divalent (FIG. 1B), but monovalent ions can play the same role at high salt concentrations. This illustrates the importance of salt in protecting RNA molecules from thermal degradation, thus allowing their activity to be maintained.

Polymerization of standard nucleotides to yield RNA-like polymers is possible in the presence of hypersaline (ammonium chloride) hydrothermal conditions (Da Silva et al. 2015). Further investigations into wet-dry cycles that generate high-salt concentrations, are needed to understand more about the polymerization mechanisms at work as well as the characteristics of the oligomers involved (Mamajanov et al. 2014).

a

While polymerization and replication of RNA is critical to understanding the origins of the RNA-world hypothesis, it is equally important to investigate the activity and functions of synthesized RNA oligomers. This is a subject that has only recently been considered. To gain further insight into the biochemical properties of RNA molecules, we studied the stability of such molecules selected in vitro at high 
temperature in the presence of salt using the SELEX (systematic evolution of ligands by exponential enrichment) method. This in vitro selection allows nucleic acid ligands called aptamers [short nucleic acid oligomers (DNA or RNA) with specific 3D shapes) to evolve and develop new functionalities. The process involves a repetitive in vitro process of sequential selection and amplification steps from a combinatorial library of nucleic acid ligands.

The SELEX method, again in the context of salty environments, helped isolate RNA molecules that were resistant to high temperatures $\left(80^{\circ} \mathrm{C}\right.$ for $\left.65 \mathrm{~h}\right)$ and high salt concentration $(2 \mathrm{M} \mathrm{NaCl})$. The sequences of the resulting cloned halo-thermophilic RNAs could be grouped in two families (I and II), each of which possesses very different thermal and chemical stabilities and very different secondary structures. The selected RNA molecules illustrate two different pathways leading to thermal resistance, which may be related to early Earth primitive conditions. It was demonstrated that members of family I constitute a good means of storing sequence information. Family II members are chemically less stable and less efficient than those of family I, but this disadvantage is compensated for, in part, during the early steps of in vitro selection in which family II were observed to replicate faster during the early cycles of SELEX. Thus, the thermal and chemical stability and the interactions of RNA with transcriptases and polymerases could be clues to help understand the evolutionary rules of RNA molecules in living organisms. Such rules could help demonstrate that what is at stake for RNA molecules, as also for living organisms, is not only "survival" but also efficient "reproduction" (Vergne et al 2006).

\section{Recent Studies on RNA Performances in Extreme and/or Hydrothermal Conditions}

The discovery of ribozymes at the beginning of the 1980s radically changed the field of studies on the origins of life by revealing the existence of modern RNAs that could have been preceded by a more ancient RNA world (Gilbert 1986). It is well-known today that RNA is responsible for the storage and transfer of genetic information, for the catalysis of some biochemical reactions, and for several regulatory and metabolic roles within the cell. The challenge now is to understand how efficient, 
functional ribozyme motifs were synthesized in prebiotic conditions. Ribozymes are stable under high temperature $\left(80^{\circ} \mathrm{C}\right.$ ) (El Murr et al. 2012) and high pressure conditions (250 MPa) (Tobé et al. 2005), and RNA is resistant to high temperatures and to high salt concentrations (4M NaCl or $4 \mathrm{M} \mathrm{KCl}$ ). Furthermore, the influence of high pressure (up to $200 \mathrm{MPa}$ ) on the reaction rate is fully reversible, meaning that the dynamics of RNA sub-conformations might have been important to catalyze various reactions during the early development of life.

\section{Sulfur and Iron-Sulfur-based Metabolism}

Apart from the polymerization and replication of RNA, the emergence of metabolism for life to sustain itself must also be considered. In very broad terms, anabolism (the constructive phase of metabolism) consists of coupled redox reaction chains that supply the energy needed to condense monomers to yield the long polymers that are required for maintaining and replicating the cell. The breakdown of long biomolecules to release energy for maintaining the cell also relies on redox reaction networks, such as those created between, dissolved inorganic ions and complexes, ion-organic molecule complexes and mineral surfaces (especially those of sulfur and iron sulfides) .

Primitive metabolic mechanisms may have employed thiol amino acids (Maurel and Orgel 2000). Thioacids are prebiotically plausible because they can be obtained inorganically by the hydrolysis of nitriles in the presence of $\mathrm{H}_{2} \mathrm{~S}$ and are very stable at neutral $\mathrm{pH}$. The oxidation of thioacids is very easy in the presence of prebiotic agents such as ferricyanide. Thus, thioacids are realistic prebiotic compounds susceptible of being active in many terrestrial environments such as the flanks of volcanoes or at oceanic hydrothermal vents.

Several theories also stress the importance of sulfur and iron in prebiotic processes. Wächtershäuser (1988) proposed that, due to the low redox potential of the $\mathrm{FeS} / \mathrm{FeS} 2$ redox system, simple prebiotic molecules such as $\mathrm{CO}_{2}$ or formaldehyde could have been reduced to more complex organic molecules. Clays or metal sulfurs from hot springs might have assisted in the production of key biochemical molecules, and the iron sulfide reactions might be the link between geochemistry and biochemistry. 
The hypothesis proposed by Wächtershäuser (1988) is referred to as the chemoautotrophic theory of the origin of life. It is based on the formation of a mineral surface, such as pyrite $\left(\mathrm{FeS}_{2}\right)$, that has been derived from iron monosulfide (FeS) and hydrogen sulfide $\left(\mathrm{H}_{2} \mathrm{~S}\right)$. Organic molecules containing negative charges are then attracted to the 2-D surface of pyriteIn an acidic geochemical solution, which was likely for early Earth, pyrite has a positive surface charge and can strongly bind anionic carboxylate and phosphate molecules, as well as mercaptochemical moieties. All these functional groups are essential in current cellular metabolisms. Bio-molecules possessing such functional groups might be the molecular ghosts of ancient molecules: the initiators of primeval functions.

According to Wächtershäuser (1988), the first organism was a chemoautotroph. Such an organism uses the formation of pyrite as a source of electrons and of energy. Carbon dioxide from the atmosphere is reduced by hydrogen, resulting in organic molecules such as carbohydrates, sugars and monomers, all of which can then lead to amino acids.

Finally, we note that some metals that are associated with protein function, such as Fe-S proteins, and of nucleic acid function, may be the evolutionary remains of ancient metals present on Earth when we think life originated (Belmonte and Mansy 2016 this issue).

\section{PRE-RNA WORLD AND GENETIC SYSTEMS}

\section{Alternative Genetic Systems (AGS)}

The difficulty in synthesising nucleotide monomers, not to mention the idea that RNA itself is too complex to have been the first genetic molecule, has focused researchers to search for non-standard molecules as earlier replicators and catalysts and on other means of transitioning to standard nucleic acids. This search has given rise to what is known as alternative genetic systems (AGSs), which attempt to model the first Darwinian ancestors, some of which are presumed to have been endowed with replicative properties (Joyce et al. 1987). One such AGS is the idea that peptide nucleic acids (PNAs) might have preceded RNA nucleotides: in PNAs, the phosphoribosyl backbone is substituted by a pseudo-peptide backbone (see Nucleotide Monomer Synthesis section). Interestingly, the PNA skeleton of N-(2- 
aminoethyl) glycine has recently been discovered in modern cyanobacteria (Banack et al. 2012). These latter authors assumed that this type of PNA skeleton might be the vestige of an ancient AGS made up of peptide-bearing nitrogenous bases. A potential transition to modern genetic systems might have been easy in protocells.

\section{RNA-Peptide World}

The origin of the genetic code and of controlled protein synthesis by the genome sequence remains one of the most poorly understood stages in the origin of life. A number of studies have shown that interactions occur between certain amino-acids or small peptides and mono- or polynucleotides. Some models suggest that the genetic code results from some specific modes of interaction between RNA and amino acids (Yarus et al. 2009): RNA can fold in such a way as to bind a specific amino acid (FIG. 2A), while proteins can expose cavities to bind a particular nucleotide (FIG. 2B).

\section{Over the Ages, Could RNA have Existed Alone?}

In order to question the plausibility of the RNA world, one might ask if we know of current molecular "living fossils," i.e. entities which represent vestiges of the RNA world. For example, at the frontiers of life we know about RNA viruses, viroids and satellite RNAs. Some viroids can behave like catalytic RNAs; hence, they are considered as remnants of the RNA world (Diener 1989).

A viroid is an RNA that is 246 to 475 nucleotides long and that folds to form rod-like structures with nucleotide base pairing (in which A pairs with $\mathrm{U}$, and $\mathrm{C}$ pairs with G). Viroids, found in nature as parasites in plant cells, are structurally, functionally and evolutionarily different from viruses. The origin of viroids is completely unknown, as is the origin of viruses. Among the main hypotheses regarding virus origins is the so-called "escape hypothesis" which states that viruses arose from genetic elements moving between cells.A second hypothesis is the "degeneracy (or regressive) hypothesis" which states that viruses are the remnants of cellular organisms. Neither of these two hypotheses can be applied to viroids.

Viroids themselves adopt a circular form, which makes them very resistant to degradation. Some viroids contain a ribozyme motif that has been termed the 
hammerhead ribozyme (HHR), which can cleave itself through strand scission. The viroid genome is small, but it can adopt a higher-order organization, something that is a known driving force in the emergence of life and molecular evolution.

The multiplication of viroids requires several catalytic activities to be accomplished: RNA polymerization, RNA cleavage and RNA ligation. RNA polymerization and RNA ligation require protein enzymes from the host plant, whereas RNA cleavage is achieved by the HHR motif of the viroid. The two missing catalytic activities from viroids have been discovered in artificially evolved RNA molecules. The molecules are an RNA-polymerase ribozyme (FIG. 3A) and two structurally divergent RNAligase ribozymes (FIG. 3C), both of which were revealed by the SELEX method.

The widespread distribution of two different families of ribozymes (hammerhead and twister) in all domains of life (bacteria, archaea and eukarya) supports the hypothesis that ribozymes were present in all cellular organisms, even early on. For a long time, circular RNAs were considered a rare cellular species or exclusive to viruses, viroids or archaea. However, circular RNAs are now recognized to be highly abundant, conserved and occur naturally in eukaryotes (Diener 1989; Lasda and Parker 2014). Finally, the viroids, and the hammerhead viroids in particular, exhibit the highest mutation rates for any biological entity. This is an important property for fast evolution (Gago et al. 2009).

The "viroid-first" hypothesis, whereby it was viroids that first appeared on Earth (albeit in an RNA world continuum), must be further experimentally and phylogenetically investigated. This is but one of many exciting avenues of research waiting to be pursued by those interested in the origin of life itself.

\section{REFERENCES}

Akouche $\mathrm{M}$ and 5 coauthors (2016) Thermal behavior of D-ribose adsorbed on silica: effect of inorganic salt coadsorption and significance for prebiotic chemistry. Chemistry 24: 15834-15846

Banack S and 5 coauthors (2012) Cyanobacteria produce N-(2-aminoethyl)glycine, a backbone for peptide nucleic acids which may have been the first genetic molecules for life on Earth. PLoS ONE 7:

http://dx.doi.org/10.1371/journal.pone.0049043 
Burcar BT and 5 coauthors (2013) Potential pitfalls in MALDI-TOF MS analysis of abiotically synthesized RNA oligonucleotides. Origins of Life and Evolution of Biospheres 43: 247-261

Britvin SN, Murasko MN, Vapnik Y, Polekhovsky YS, Krivovichev SV (2015) Earth's phosphides in Levant and insights into the source of archean prebiotic phosphorus. Scientific Reports 5: doi:10.1038/srep08355

Da Silva L, Maurel M-C, Deamer D (2015) Salt-promoted synthesis of RNA-like molecules in simulated hydrothermal conditions. Journal of Molecular Evolution 80: 86-97

Diener TO (1989) Circular RNAs: relics of precellular evolution? Proceedings of the National Academy of Sciences of the United States of America 86: 9370-9374

Ferris JP, Hill AR Jr, Liu R, Orgel LE (1996) Synthesis of long prebiotic oligomers on mineral surfaces. Nature 381: 59-61

Ferris JP (2005) Mineral catalysis and prebiotic synthesis: montmorillonitecatalyzed formation of RNA. Elements 1: 145-149

Gago S, Elena SF, Flores R, Sanjuán R (2009) Extremely high mutation rate of a hammerhead viroid. Science 323: 1308

Georgelin T and 6 coauthors (2015) Stabilisation of ribofuranose by a mineral surface. Carbohydrate Research 402: 241-244

Gilbert W (1986) Origin of life: The RNA world. Nature 319: 618

Hill AR Jr, Böhler C, Orgel LE (1998) Polymerization on the rocks: negativelycharged $\alpha$-amino acids. Origins of Life and Evolution of Biospheres 28: 235243

Joyce GF, Schwartz AW, Miller SL, Orgel LE (1987) The case for an ancestral genetic system involving simple analogues of the nucleotides. Proceedings of the National Academy of Sciences of the United States of America 84: 4398-4402

Lasda E, Parker R (2014) Circular RNAs: diversity of form and function. RNA 20: 1829-1842

Leclerc F and 5 coauthors (2016) Self-assembly controls self-cleavage of HHR from ASBVd(-): a combined SANS and modeling study. Scientific Reports 6: doi:10.1038/srep30287

Mamajanov I and 10 coauthors (2014) Ester formation and hydrolysis during wetdry cycles: generation of far-from-equilibrium polymers in a model prebiotic reaction. Macromolecules 47: 1334-1343

Maurel M-C, Ninio J (1987) Catalysis by a prebiotic nucleotide analog of histidine. Biochimie 69: 551-553

Maurel M-C, Orgel LE (2000) Oligomerization of alpha-thioglutamic acid. Origins of Life and Evolution of Biospheres 30:423-430

Meinert C and 7 coauthors (2016) Ribose and related sugars from ultraviolet irradiation of interstellar ice analogs. Science 352: 208-212 
Miller SL (1987) Which organic compounds could have occurred on the prebiotic Earth? Cold Spring Harbour Symposia on Quantitative Biology 52: 17-27

El-Murr N and 6 coauthors (2012) Behavior of a hammerhead ribozyme in aqueous solution at medium to high temperatures. Naturwissenschaften 99: 731-738

Paecht-Horowitz M (1978) The influence of various cations on the catalytic properties of clays. Journal of Molecular Evolution 11: 101-107

Ricardo A, Carrigan MA, Olcott AN, Benner SA (2004) Borate minerals stabilize ribose. Science 303: 196

Saladino R, Crestini C, Ciciriello F, Costanzo G, Di Mauro E (2007) Formamide chemistry and the origin of informational polymers. Chemistry and Biodiversity 4: 694-720

Szostak JW (2012) The eightfold path to non-enzymatic RNA replication. Journal of Systems Chemistry 3: 1-14

Tkachenko AV, Maslov S (2015) Spontaneous emergence of autocatalytic information-coding polymers. Journal of Chemical Physics 143: 045102

Tobé S, Heams T, Vergne J, Hervé G, Maurel M-C (2005) The catalytic mechanism of hairpin ribozyme studied by hydrostatic pressure. Nucleic Acids Research 33: $2557-2564$

Vergne J, Cognet JAH, Szathmáry E, Maurel M-C (2006) In vitro selection of halothermophilic RNA reveals two families of resistant RNA. Gene 371: 182-193

Wächtershäuser G (1988) Before enzymes and templates: theory of surface metabolism. Microbiological Reviews 52: 452-484

Yarus M, Widmann JJ, Knight R (2009) RNA-amino acid binding: a stereochemical era for the genetic code. Journal of Molecular Evolution 69: 406-429

\section{FIGURE CAPTIONS:}

SCHEME 1 Chemical structure of alanine-adenylate. The energy-rich bond between the carboxyl and the phosphate group is indicated in red.

FiguRE 1 Canonical structures of transfer RNA (tRNA) and the role of tertiary contacts (remote paired nucleotides) and metal ions in the stabilization of RNA. (A) Cloverleaf secondary structure: Blue - paired regions; yellow - unpaired regions; brown and green lines - tertiary contacts established between different unpaired regions from different domains of the tRNA. (B) L-shape 3-D structure of tRNA; colour code same as for $1 \mathrm{~A}$; purple spheres - metal ions $\mathrm{Mg}^{2+}$ or $\mathrm{Mn}^{2+}$. The 2-D and 3-D structures correspond to the tRNA-Phe molecule (PDB ID: 1EHZ). 
FIGURE 2 RNA/amino acid and nucleotide/protein interactions as a potential relict of the primordial genetic code. (A) Interaction between a lysine amino acid and a riboswitch. The amino acid is shown using an atomic CPK (Corey-Pauling-Koltun) model and the nucleotides from the RNA riboswitch by a stick model (the RNA chain is shown by a purple ribbon). The charged amino acid interacts simultaneously with several nucleotide residues of the RNA. Hydrogen bond contacts are indicated by dashed lines (PDB ID: 3D0U). (B) Interaction between a nucleotide residue (adenosine) from an RNA chain and a human protein (PUMILIO1). The nucleotide is shown using an atomic CPK model and the surrounding protein residues by a stick model. The nucleotide residue interacts simultaneously with several amino-acid residues (PDB ID: 1M8W)

FIGURE 3 Structures of artificial and natural ribozymes with the three basic catalytic activities required for viroid replication. (A) RNA-polymerase ribozyme (PDB ID: 3IVK); the 3-D structure corresponds to a class I RNA ligase (first chemical step of RNA polymerization); a fully active RNA-polymerase ribozyme (capable of catalyzing the accurate synthesis of an RNA sequence longer than itself) adopts the same 3-D fold. (B) Hammerhead ribozyme (PDB ID: 20EU) with an RNase-like activity caused by self-cleaving. (C) RNA ligase ribozyme (PDB ID:2OIU). The small coloured dots in and around the three structures indicate the presence of tightly bound metal ions and water molecules.

NOTE: In Fig 3, the letters A, B, and C appear on my screen as slightly distorted. Please check if this tallies with your screen and if so, please make neater. 


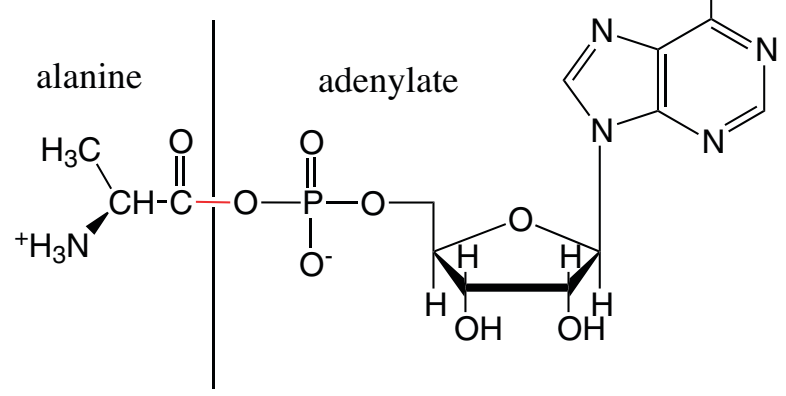

SCHeme 1 Chemical structure of alanine-adenylate. The energyScheme 1 rich bond between the carboxyl and the phosphate group is indicated in red.
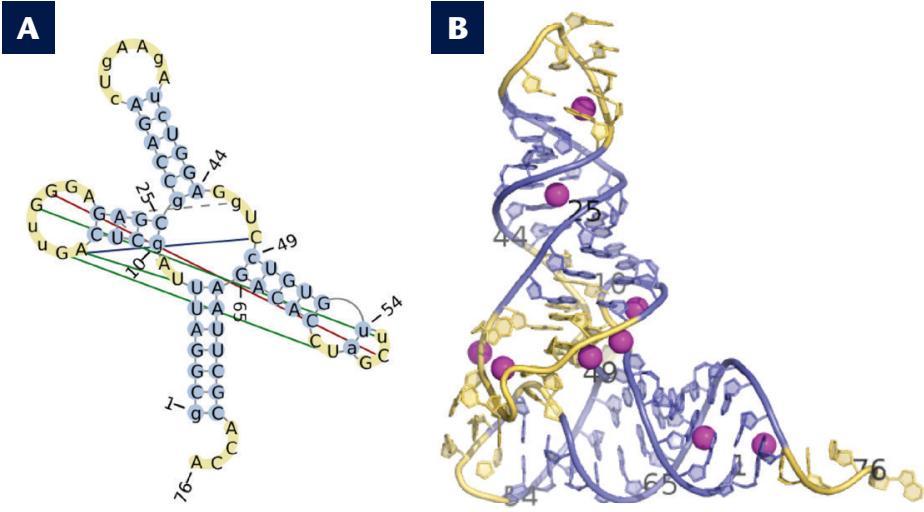

Figure 1 Canonical structures of transfer RNA (tRNA) and the le of tertiary contacts (remote paired nucleotides) and metal ions in the stabilization of RNA. (A) Cloverleaf secondary structure: Blue - paired regions; yellow - unpaired regions; brown and green lines - tertiary contacts established between different unpaired regions from different domains of the tRNA. (B) L-shape 3-D structure of tRNA; colour code same as for $1 \mathrm{~A}$; purple spheres - metal ions $\mathrm{Mg}^{2+}$ or $\mathrm{Mn}^{2+}$. The 2-D and 3-D structures correspond to the tRNA-Phe molecule (PDB ID: 1EHZ). 


\section{A}
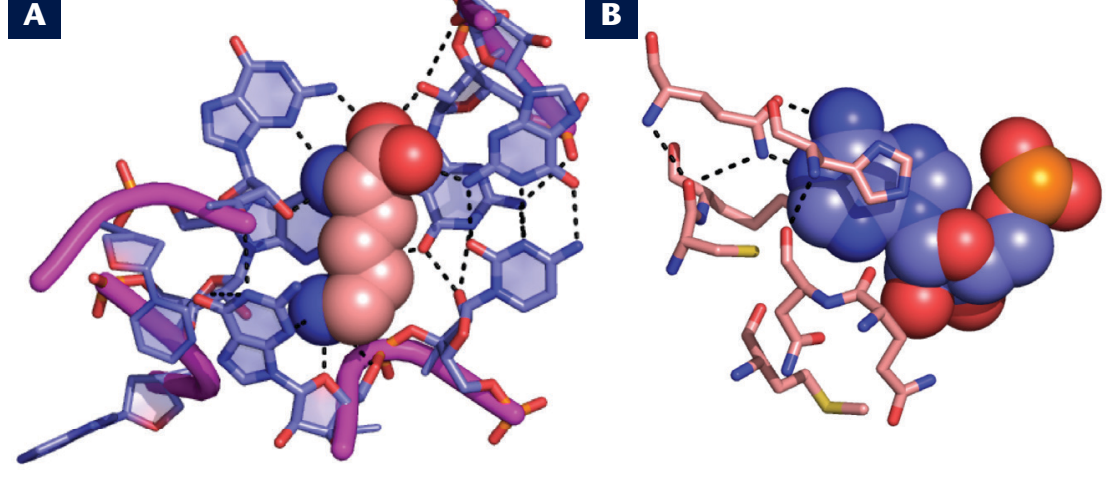

Figure 2 RNA/amino acid and nucleotide/protein interactions

as a potential relict of the primordial genetic code.

(A) Interaction between a lysine amino acid and a riboswitch. The amino acid is shown using an atomic CPK (Corey-Pauling-Koltun)

model and the nucleotides from the RNA riboswitch by a stick

model (the RNA chain is shown by a purple ribbon). The charged

amino acid interacts simultaneously with several nucleotide residues

of the RNA. Hydrogen bond contacts are indicated by dashed lines

(PDB ID: 3D0U). (B) Interaction between a nucleotide residue

(adenosine) from an RNA chain and a human protein (PUMILIO1).

The nucleotide is shown using an atomic CPK model and the

surrounding protein residues by a stick model. The nucleotide

residue interacts simultaneously with several amino-acid residues

(PDB ID: $1 \mathrm{M} 8 \mathrm{~W}$ ) 


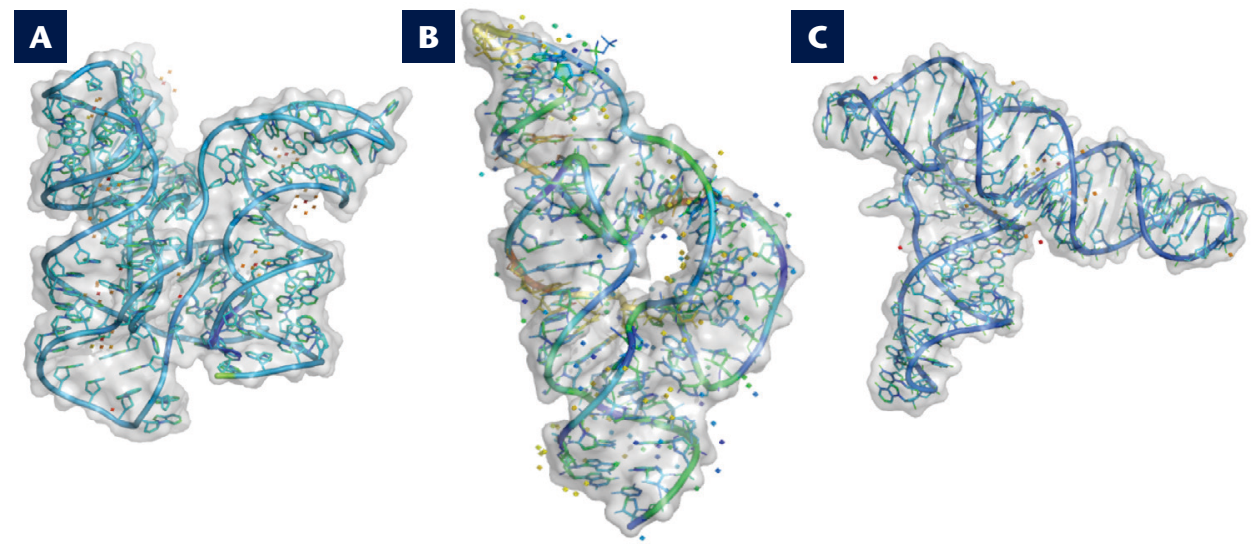

Figure 3 Structures of artificial and natural ribozymes with the cation. (A) RNA-polymerase ribozyme (PDB ID: 3IVK); the 3-D structure corresponds to a class I RNA ligase (first chemical step of RNA polymerization); a fully active RNA-polymerase ribozyme (capable of catalysing the accurate synthesis of an RNA sequence longer than itself) adopts the same 3-D fold. (B) Hammerhead ribozyme (PDB ID: 2OEU) with an RNase-like activity (self-cleaving). (C) RNA ligase ribozyme (PDB ID:2OIU). The small coloured dots in and around the three structures indicate the presence of tightly bound metal ions and water molecules. 